\title{
Study of retrograde conduction in complete heart block using His bundle recordings
}

\author{
Akira Takeshita, Senichi Tanaka, and Motoomi Nakamura \\ From the Research Institute of Angiology and Cardiovascular Clinic, Kyushu University Hospitals, Fukuoka \\ fapan
}

Two patients with complete anterograde AV block without retrograde $P^{\prime}$ waves are described in whom regular retrograde conduction across the blocked area was shown by recording the His bundle electrogram. When an atrial depolarization $(A)$ occurred after the $Q R S$ complex within a certain period, $A$ was followed either by no $H$ potential or by a prolonged $A H$ interval, indicating concealed retrograde penetration of the His bundle. The fact that retrograde conduction affected anterograde conduction indicates that the anterograde and retrograde pathways are not totally independent. Atropine significantly improved retrograde conduction; this suggests that the retrograde conduction occurs through the conduction system in a retrograde fashion rather than through a muscular pathway, bypassing a blocked area of the conduction system, as the latter should not be affected by atropine.

It is well known that the conduction system is capable of transmitting impulses in both an anterograde and retrograde fashion (Moe, Preston, and Burlington, 1956; Rosenblueth, 1958). Failure to transmit impulses in one direction does not necessarily mean that conduction in the opposite direction is also blocked. Retrograde ventriculoatrial conduction has been known to occur even in the presence of complete block of anterograde atrioventricular conduction (Wolferth and McMillan, 1929; Winternitz and Langendorf, 1944; Scherf, Cohen, and Orphanos, 1964; Gubbay and Mora, 1964; Louvros and Costeas, 1965; Castillo and Samet, 1967; Gupta and Haft, 1972).

Various theories have been given to explain the mechanism of retrograde conduction in the presence of complete anterograde block. Some investigators have postulated that separate pathways might exist for anterograde and retrograde conduction (Danielopolu and Danulescu, 1922). The possibilities that pacemaker cells in the conduction system above the blocked area might be stimulated mechanically (Barker, 1925), or by an electrotonic spread of the action potentials across the blocked area (Scherf, 1959) have been suggested. The concepts of supernormal conduction (Kline, Conn, and Rosenbaum, 1939) or decremental conduction (Bellet, I97I) have also been used to explain this phenomenon.

Received 26 October 1973.
Recordings of His bundle electrograms have been used extensively for studying the mechanisms of various arrhythmias (Damato et al., 1969; Narula et al., I971; Rosen et al., 1970; Castellanos et al., 1970; Goldreyer and Damato, 1971), but few studies only have been performed in which the His bundle electrogram was recorded to elicit the mechanisms of retrograde conduction in the presence of complete AV block (Gupta and Haft, 1972). This report presents two cases with complete AV block in which the presence of $I: I$ retrograde conduction was shown by the His bundle electrogram even in the absence of retrograde $P^{\prime}$ waves. The evidence suggesting that anterograde and retrograde conduction pathways are not totally independent is presented.

\section{Case I}

\section{Case reports}

A 64-year-old man was admitted with a history of fainting, syncope, and mild dyspnoea on exertion of a few months' duration. One month before admission the electrocardiogram showed Mobitz type II AV block. On physical examination, the pulse was regular at a rate of 34 a minute and the blood pressure was $150 / 78 \mathrm{mmHg}$. Cardiac examination revealed a first heart sound of varying intensity, occasional fourth heart sound, but no murmur. The electrocardiogram showed complete AV block with an atrial rate of 58 a minute, and a ventricular rate of 40 a minute. The chest $x$-ray showed a heart of normal size. The electrocardiogram at times 
showed Mobitz type II AV block with complete right bundle-branch block and a prolonged PQ interval of 0.22 sec.

\section{Case 2}

A 48-year-old man was admitted with an episode of syncope two months before admission. He did not give a history of dyspnoea on exertion or angina pectoris. On physical examination, the pulse was regular at a rate of 40 a minute and the blood pressure was $160 / 78 \mathrm{mmHg}$. Examination of the heart showed occasional cannon first heart sounds with an ejection systolic murmur of grade 2/6 at the apex. The electrocardiogram showed complete AV block, with an atrial rate of 88 a minute and a ventricular rate of 40 a minute. No cardiac enlargement was seen on the chest $x$-ray.

\section{Electrophysiological studies}

Electrophysiological studies were performed at the time of cardiac catheterization. His bundle electrograms (HBE) were recorded with a tripolar catheter passed to the right side of the heart, using a modification of the method of Damato et al. (1969). Recordings were obtained at a paper speed of $100 \mathrm{~mm} / \mathrm{sec}$ on a multichannel oscilloscopic photographic recorder (Hewlett Packard 4568B). Atrial and ventricular pacing were accomplished with a bipolar electrode catheter placed against the lateral wall of the right atrium and at the apex of the right ventricle, respectively.

Analysis of $\mathrm{His}$ bundle electrograms showed the following.

I) Control recordings before administration of atropine in both patients showed complete anterograde AV

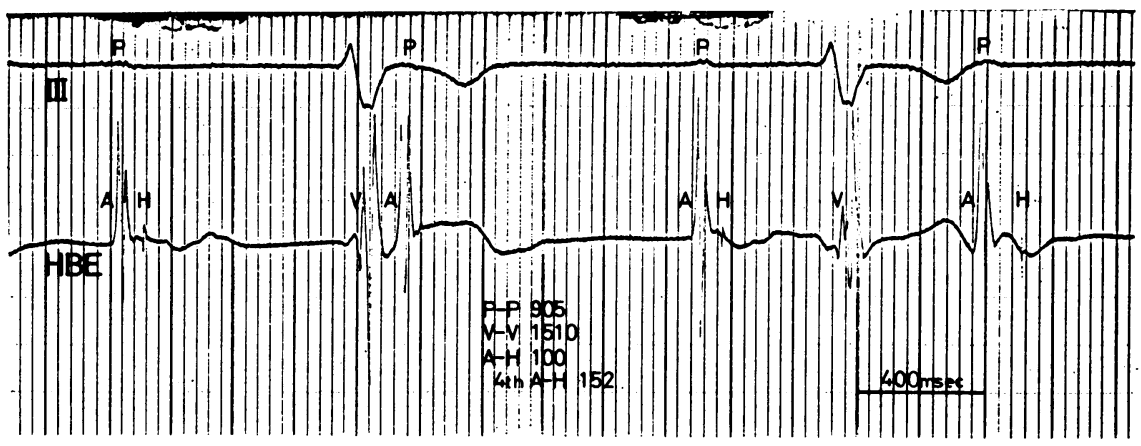

FIG. I His bundle recording showing concealed retrograde conduction in Case I. Lead III of the electrocardiogram and His bundle electrogram (HBE). $P$ waves are labelled $P$ in lead III. An atrial, bundle of $H i$, and ventricular potential on $H B E$ are labelled $A, H$, and $V$, respectively. Complete $A V$ dissociation is present, with an atrial rate of 66 a minute and a ventricular rate of 40 a minute. AV block occurs after the $H$ potential. The basic $A H$ interval is normal and constant (100 msec, the first and third $A H$ ). The second $A$ is not followed by $H$ when $A$ occurred only $190 \mathrm{msec}$ after $V$. The fourth $A H$ interval is prolonged $(152 \mathrm{~mm} / \mathrm{sec})$ when $A$ occurred $482 \mathrm{msec}$ after V. Paper speed is $100 \mathrm{~mm} / \mathrm{sec}$ in this and subsequent illustrations.

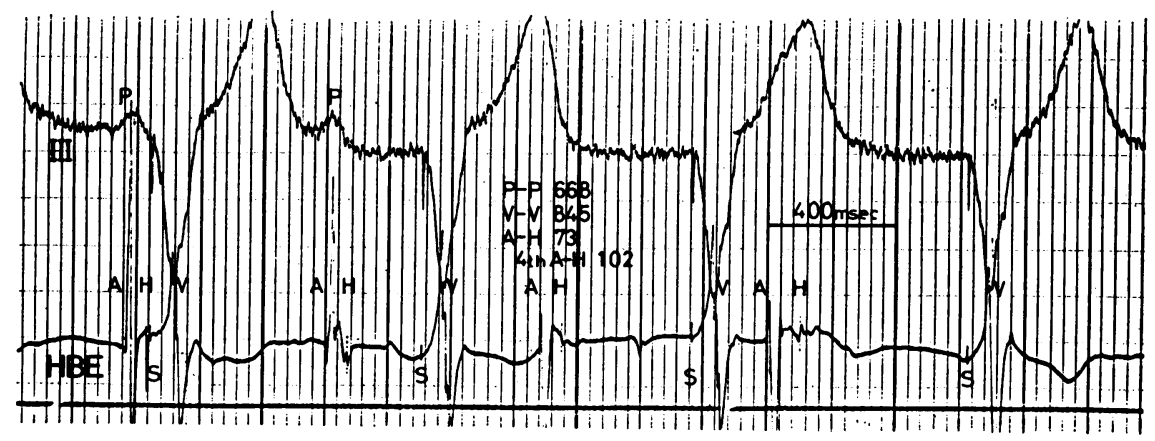

FIG. 2 His bundle recording showing concealed retrograde conduction in Case 2. $S$ indicates spikes of ventricular stimulation. AH is normal and constant $(73 \mathrm{msec}$ ) except for the fourth $A H$ (I02 $\mathrm{msec}$ ) when A occurred only $240 \mathrm{msec}$ after $V$. 


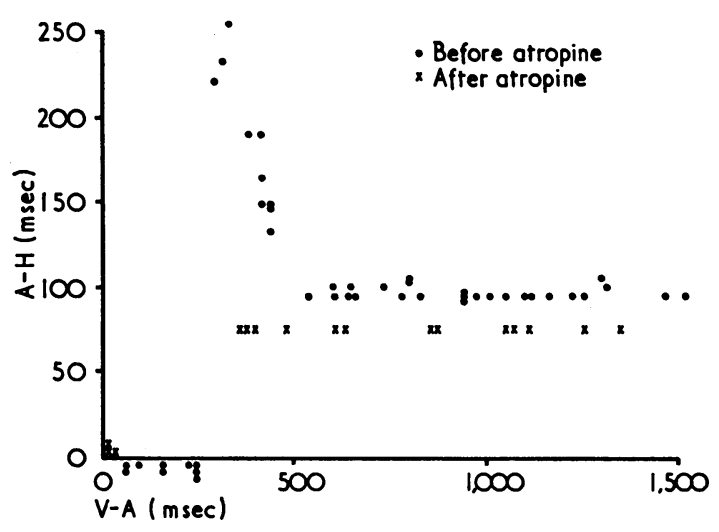

FIG. 3 Effect of the interval of $V$ to $A$ on the following $A H$ interval before and after atropine in Case I. When $A$ occurred less than $460 \mathrm{msec}$ after $V, A H$ was always prolonged. The shorter the $V$ to $A$ interval, the longer the $A H$ interval. No $H$ followed $A$ when the $V$ to $A$ interval was less than $295 \mathrm{msec}$. After atropine no prolongation of $A H$ was seen even when $A$ occurred less than 460 msec after $V$.

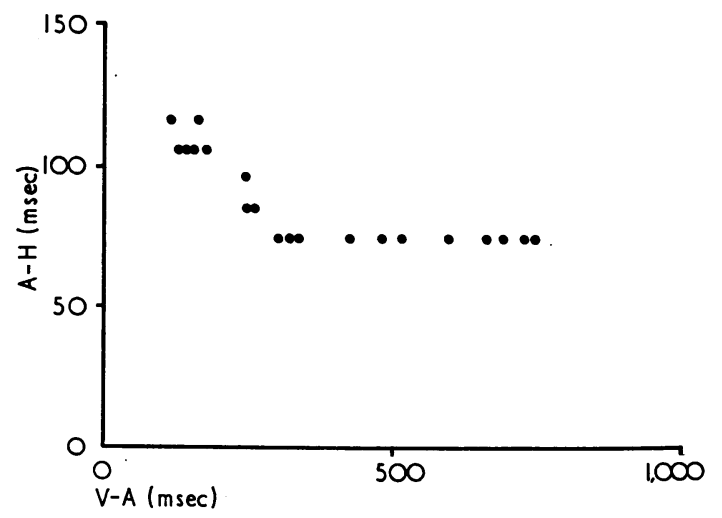

FIG. 4 Effect of the interval of $V$ to $A$ on the following $A H$ interval before atropine in Case 2. $A H$ was always prolonged when $A$ occurred less than 279 msec after $V$.

block with block distal to the $\mathrm{H}$ potential. The PP and $R R$ intervals were constant in both patients, i.e. 905 $\mathrm{msec}$ and $1510 \mathrm{msec}$, respectively in Case $\mathrm{I}$, and 618 msec and $1360 \mathrm{msec}$ in Case 2. No retrograde $\mathrm{P}^{\prime}$ wave was seen in either patient.

2) When an atrial depolarization (A) came soon after the ventricular complex (V), A was followed by either absence of $\mathrm{H}$ potential or prolongation of the $A$ to $H$ interval (Fig. I and Fig. 2). This suggests that the AV node or bundle of His was depolarized in a retrograde fashion by the preceding ventricular complex and hence was refractory to anterograde depolarization. In both patients the presence of $I: I$ retrograde conduction was suggested by the finding that the AH interval was always prolonged when $A$ came after $V$ within a certain period. When A came after V within $460 \mathrm{msec}$ in Case $r$, it was noted that the shorter the interval between $\mathrm{V}$ and $\mathrm{A}$, the longer was the following AH interval (Fig. 3).

$A$ similar relation between the interval of $V$ to $A$ and the following $\mathrm{AH}$ interval was noted in Case 2, but it occurred only at a considerably shorter VA interval than in Case I (Fig. 4).

3) After intravenous administration of $\mathrm{I} \mathrm{mg}$ atropine in Case $\mathrm{I}$, no prolongation of the $\mathrm{AH}$ interval was observed even when A came after $V$ within $460 \mathrm{msec}$, and occasional retrograde $\mathrm{P}^{\prime}$ waves were seen (Fig. 5). These observations indicate that atropine increases conductivity for retrograde conduction and suggest that velocity of retrograde conduction was increased by atropine so that the AV node or bundle of His was recovered fully by the time the next anterograde impulse came through.

4) Retrograde conduction to the atrium, producing a retrograde $P^{\prime}$ wave, was observed only when $V$ occurred between $300 \mathrm{msec}$ and $440 \mathrm{msec}$ after A (Fig. 6). When A preceded $\mathrm{V}$ by more than $580 \mathrm{msec}$, the next sinus $\mathrm{P}$ wave occurred right after $\mathrm{V}$ before retrograde conduction could reach the atrium. Presence of retrograde conduction to the AV node or bundle of His was indicated by the absence of an $\mathrm{H}$ potential following this sinus $\mathrm{P}$ wave. When $V$ came sooner than $220 \mathrm{msec}$ after $\mathrm{A}$, no retrograde conduction was seen. This may be explained either by the presence of concealed anterograde conduction beyond the bundle of His, which could then make the area refractory to the next retrograde impulse, or by long refractoriness of the AV node or bundle of His to retrograde conduction. Since the retrograde $\mathrm{H}$ potential is buried in the QRS complex, the exact site of retrograde block cannot be known.

\section{Discussion}

As early as 1914, Cohn and Fraser observed the abnormal $\mathrm{P}$ waves in heart block for the first time in man. In 1929, Wolferth and McMillan observed abnormal $\mathbf{P}$ waves in complete AV block and concluded that this was due to retrograde conduction. This subject was critically reviewed by Winternitz and Langendorf in 1944 and since then a few case reports have been published (Scherf et al., 1964; Gubbay and Mora, 1964; Louvros and Costeas, 1965).

Although Castillo and Samet (1967) found retrograde conduction to the atrium in as many as 4 out of 7 patients with complete AV block, most studies indicate that retrograde conduction occurs uncommonly in patients with anterograde AV block. Goldreyer and Bigger studied retrograde VA conduction in 50 patients, 24 of whom had impairment of anterograde AV conduction. Only 2 out of 24 patients with any degree of AV block and only one 

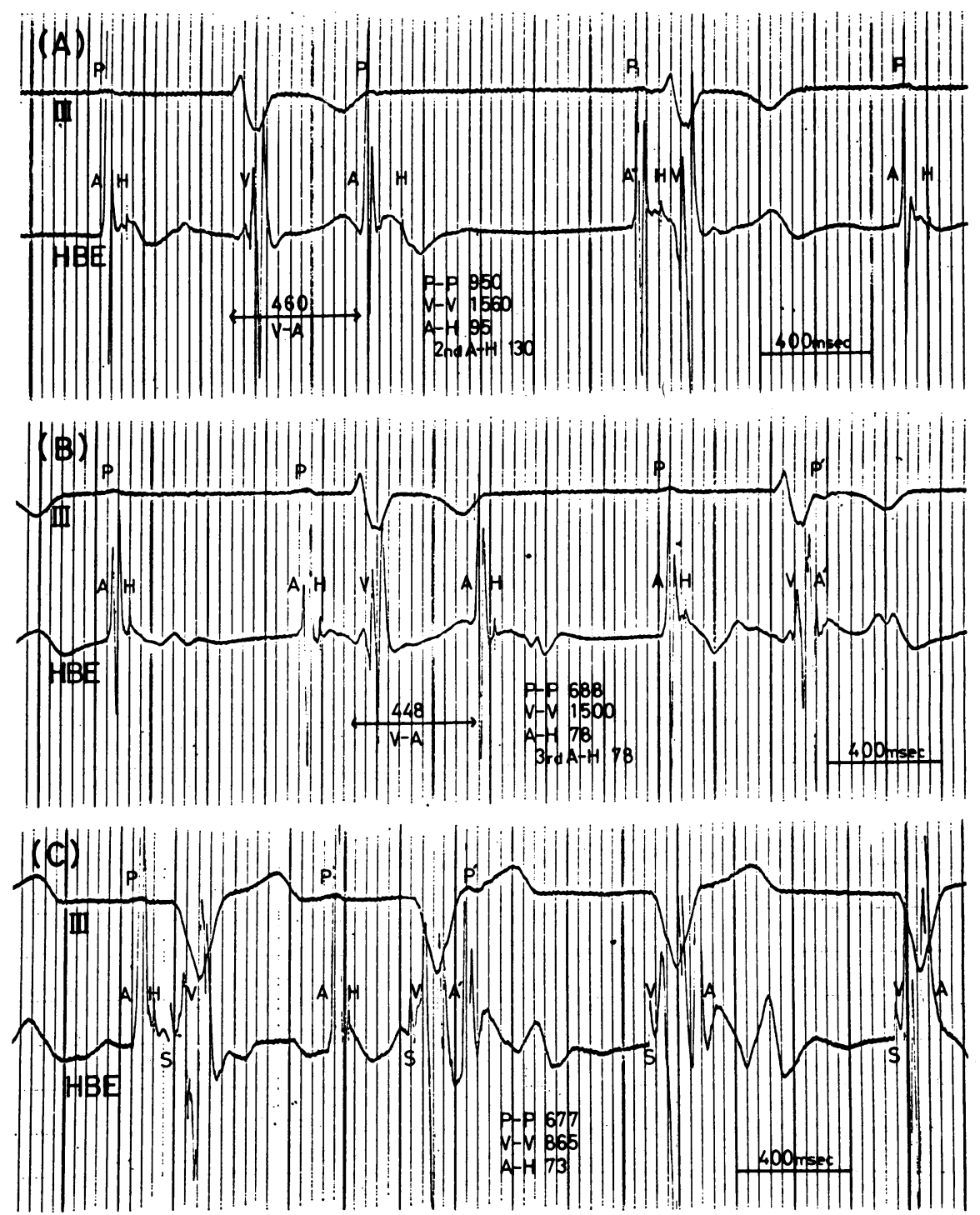

FI G. 5 Effect of atropine retrograde conduction in Case 1. $A$ ) Before atropine. The second $A H$ is prolonged when $A$ occurred only $460 \mathrm{msec}$ after $V$. PP interval is $950 \mathrm{msec}$ and the basic $A H$ interval is $95 \mathrm{msec}$. B) After atropine. Both PP and AH intervals are shortened by atropine. $P P$ is $688 \mathrm{msec}$ and $A H$ is $78 \mathrm{msec}$. The third $A H$ is not prolonged even when $A$ occurred only 488 msec after $V$. Retrograde $P^{\prime}$ wave is seen after the second $Q R S$ complex. $C$ ) Ventricular pacing after atropine. Retrograde $P^{\prime}$ and $A^{\prime}$ are shown after the second $Q R S$ complex. The fourth and fifth $A$ are anterograde sinus $A$ without $H$.

of 12 patients with complete AV block had retrograde VA conduction (Goldreyer and Bigger, 1970).

A number of theories have been postulated to explain retrograde VA conduction in the presence of complete anterograde AV block. It has been sug- gested that mechanical stimulation by ventricular contraction of the automatic cells in the conduction system above the site of the block might initiate an impulse and produce retrograde conduction (Barker, 1925; Cohn and Fraser, 1914). Separate pathways for anterograde and retrograde conduction have been 


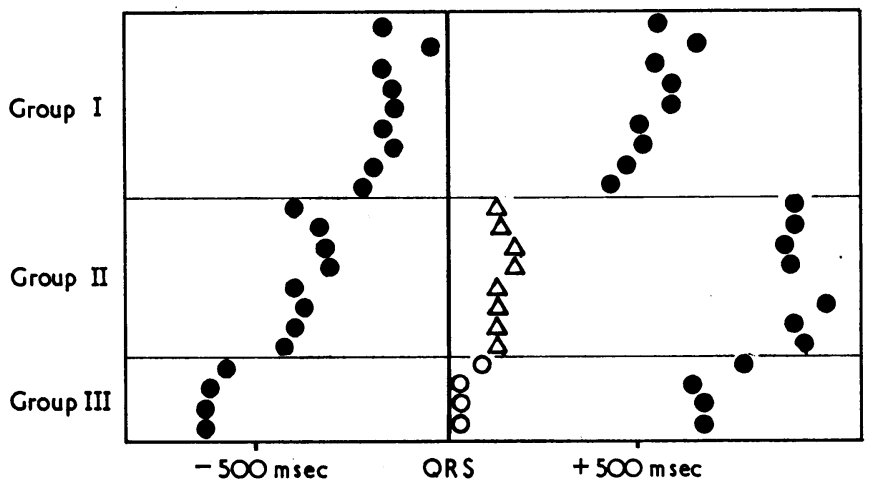

FIG. 6 Relation between the appearance of a retrograde $P^{\prime}$ wave and the interval of preceding $A$ to $Q R S$ complex $(V)$. The circles indicate $A$ waves produced by a sinus beat; solid circles represent these waves followed by an $H$ deflection and open circles indicate those waves without a succeeding $H$. The triangles indicate retrograde $P^{\prime}$ waves; these are not followed by $H$ (hence open triangles). Note that retrograde $P^{\prime}$ waves are seen only when $A$ came between 300 and 400 msec before the $Q R S$ complex. (See text for detail.)

postulated, in which destruction of the former with preservation of the latter would permit retrograde conduction in the presence of anterograde AV block (Danielopolu and Danulescu, 1922). Kline et al. (I939) suggested that a supernormal phase of conductivity is produced at the site of the block by the blocked anterograde impulse so as to allow conduction of the next ventricular beat in retrograde fashion. Scherf (1959) mentioned the possibility that an electrotonic spread of the action potential caused by the activation of the ventricle allows retrograde conduction. Decremental conduction has been invoked to explain retrograde conduction in complete AV block (Bellet, 197I). It is known that the cardiac impulse normally undergoes decrement in the AV node. According to this theory the anterograde conduction is blocked because the impulse has undergone the maximal decrement when it reaches the barrier area but a retrograde impulse may penetrate the barrier area before undergoing decrement.

Recording of His bundle electrograms in the patients with complete anterograde AV block demonstrated, I) the presence of I: I retrograde conduction across the blocked area, even without retrograde $P^{\prime}$ waves, 2) that anterograde and retrograde conduction pathways are not totally independent, and 3) that atropine increased conductivity for retrograde conduction.

The presence of $I: I$ retrograde conduction across the blocked area, which was distal to the bundle of His in the patients studied, was suggested by the fact that the $\mathrm{AH}$ interval was always prolonged when A occurred after $\mathrm{V}$ within a certain period. The observation that retrograde conduction affected anterograde AH conduction indicates that anterograde and retrograde conduction pathways are not totally independent, at least at the AV node or bundle of His. The latter observation is in agreement with the observation made by Gupta and Haft (1972), who also noted a prolonged AH interval caused by retrograde conduction in a patient with complete anterograde AV block.

Atropine increased conductivity for retrograde conduction as shown in Fig. 3 and 5 This finding is compatible with the finding of Damato, Lau, and Bobb (1970) that in dogs retrograde VA conduction was delayed by vagal stimulation.

The retrograde $\mathbf{P}^{\prime}$ wave was seen late in atrial diastole, only shortly before the next normal $P$ wave was due. The ventricular complex which produced a retrograde $P^{\prime}$ wave occurred between $300 \mathrm{msec}$ and $440 \mathrm{msec}$ after the preceding atrial depolarization. Appearance of retrograde $\mathbf{P}^{\prime}$ waves only late in atrial diastole has been described and was attributed to long refractoriness of the AV node for retrograde conduction (Scherf et al., 1964). In fact the VA effective refractory period was longer than the AV effective refractory period at similar driven cycle lengths (Goldreyer and Bigger, 1969). Another possible explanation is the presence of concealed anterograde conduction beyond the bundle of His which can make the area refractory to retrograde conduction of the next ventricular beat when it occurs soon after an atrial depolarization. However, the exact site of retrograde block is not known be- 
cause the retrograde $\mathrm{H}$ potential is buried in the QRS complex.

Although the present study shows that anterograde and retrograde conduction take the same pathway at the AV node or bundle of His, it is not clear if conduction from the ventricle to the node goes through the blocked conduction system in a retrograde fashion, or if it goes around the block through muscular conduction. Which pathway of conduction is actually used could be determined if the exact site of retrograde block could be demonstrated. However, the fact that conductivity of retrograde conduction was significantly increased by atropine suggests that muscular conduction is unlikely.

\section{References}

Barker, P. S. (1925). The occurrence of auricular beats due to stimulation of the auricles by the contracting ventricles during complete heart-block. American Heart fournal, I, 349 .

Bellet, S. (1971). Clinical Disorders of the Heart Beat, 3rd ed., p. 384. Lea and Febiger, Philadelphia.

Castellanos, A., Jr., Chapunoff, E., Castillo, C., Maytin, O., and Lemberg, L. (1970). His bundle electrograms in two cases of Wolff-Parkinson-White (pre-excitation) syndrome. Circulation, 4I, 399.

Castillo, C., and Samet, P. (1967). Retrograde conduction in complete heart block. British Heart fournal, 29, 553.

Cohn, A. E., and Fraser, F. R. (1914). The occurrence of auricular contractions in a case of incomplete and complete heart-block due to stimuli received from the contracting ventricles. Heart, $5,14 \mathrm{I}$.

Damato, A. N., Lau, S. H., and Bobb, G. A. (1970). Studies on ventriculo-atrial conduction and the reentry phenomenon. Circulation, 41, 423.

Damato, A. N., Lau, S. H., Helfant, R., Stein, E., Patton, R. D., Schlerlag, B. J., and Berkowitz, W. D. (1969). A study of heart block in man using His bundle recordings. Circulation, 39, 297.

Danielopolu, D., and Danulescu, V. (1922). Retrograde conductivity. Archives des Maladies du Coeur et des Vaisseaux, I5, 365 .

Goldreyer, B. N., and Bigger, J. T. (1969). Spontaneous and induced reentrant tachycardia. Annals of Internal Medicine, 70, 87 .
Goldreyer, B. N., and Bigger, J. T. (1970). Ventriculo-atrial conduction in man. Circulation, 41, 935.

Goldreyer, B. N., and Damato, A. N. (1971). Essential role of atrioventricular conduction delay in the initiation of paroxysmal supraventricular tachycardia. Circulation, 43, 679.

Gubbay, E. R., and Mora, C. A. (1964). Retrograde conduction and isorhythmic dissociation in heart block. American Heart fournal, 68, 166.

Gupta, P. K., and Haft, J. I. (1972). Retrograde ventriculoatrial conduction in complete heart block. Studies with His bundle electrography. American fournal of Cardiology, 30, 408.

Kline, E. M., Conn, J. W., and Rosenbaum, F. F. (1939). Variations in A-V and V-A conduction dependent upon the time relations of auricular and ventricular systole: the supernormal phase. American Heart fournal, 17, 524.

Louvros, N., and Costeas, F. (1965). Retrograde activation of atria in auriculo-ventricular block. Archives of Internal Medicine, 116, 778.

Moe, G. K., Preston, J. B., and Burlington, H. (1956). Physiologic evidence for a dual A-V transmission system. Circulation Research, 4, 357.

Narula, O. S., Scherlag, B. J., Samet, P., and Javier, R. P. (197I). Atrioventricular block: localization and classification by His bundle recordings. American fournal of Medicine, 50, 146.

Rosen, K. M., Loeb, H. S., Chuquimia, R., Sinno, M. Z., Rahimtoola, S. H., and Gunnar, R. M. (1970). Site of heart block in acute myocardial infarction. Circulation, 42, 925.

Rosenblueth, A. (1958). Two processes for auriculo-ventricular and ventriculo-auricular propagation of impulses in the heart. American fournal of Physiology, 194, 495.

Scherf, D. (1959). Retrograde conduction in complete heart block. Diseases of the Chest, 35, 320.

Scherf, D., Cohen, J., and Orphanos, R. P. (1964). Retrograde activation of atria in atrioventricular block. American fournal of Cardiology, 13, 219.

Winternitz, M., and Langendorf, R. (1944). Auriculoventricular block with ventriculoauricular response. Report of six cases and critical review of the literature. American Heart fournal, 27, 30r.

Wolferth, C. C., and McMillan, T. M. (1929). Observation on the mechanism of relatively short intervals in ventriculoauricular and auriculoventricular sequential beats during high grade heart-block. American Heart fournal, 4, 521 .

Requests for reprints to Dr. Akira Takeshita, Research Institute of Angiocardiology and Cardiovascular Clinic, Kyushu University Hospitals, Fukuoka, Japan 812. 